\title{
Diagnostik und Therapie der Varikosis
}

\section{Diagnostic and Treatment of Varicose Veins}

B. Kahle, M. Stücker

Klinik für Dermatologie, Allergologie und Venerologie, Universitätsklinikum Schleswig-Holstein

\section{Lernziele \\ $\nabla$}

Kenntnisse über:

- die Epidemiologie der Venenkrankheiten

- die primäre Varikosis

- die chronisch venöse Insuffizienz

- Diagnostik und Therapie der Varikosis

\section{Einleitung \\ $\nabla$}

Die Varikosis ist entsprechend der Leitlinien definiert als eine degenerative Erkrankung der Venenwand im oberflächlichen Venensystem der Beine, bei der sich unter dem Einfluss verschiedener Realisationsfaktoren im Laufe des Lebens in unterschiedlicher Ausprägung und Schweregrad Krampfadern (Varizen) entwickeln [1]. Nach Morphologie und Topografie werden dabei 5 Varizentypen unterschieden: Stammvarizen, Seitenastvarizen, Perforansvarizen, retikuläre Varizen und Besenreiservarizen.

\section{Epidemiologie}

Venenkrankheiten zählen zu den häufigsten Erkrankungen in der Bevölkerung. Die Prävalenz von chronischen venösen Erkrankungen sowie deren sozioökonomische Bedeutung wurden bereits in zahlreichen epidemiologischen Studien untersucht [2-6]. In der „Tübinger Studie“ [4] von 1979 an 4500 Erwachsenen konnte lediglich bei $14 \%$ kein objektivierbarer pathologischer Venenbefund erhoben werden. 58\% der Untersuchten zeigten geringe Venenveränderungen, 15\% litten an einer ausgeprägten Varikosis und 13\% an einer fortgeschrittenen chronischen venösen Insuffizienz (CVI).

Der Anteil der Probanden mit retikulären Varizen und Besenreiservarizen war mit 59\% vergleichbar mit der Rate, die in der „Tübinger Studie“ erhoben wurde. Ebenso besteht eine Übereinstimmung bezüglich der Häufigkeit der Varikosis. Die „Bonner Studie“ zeigte in 14,3\% der Fälle aus-
Aktuelle Daten der „Bonner Venenstudie“, einer breit angelegten bevölkerungsbasierten Querschnittsuntersuchung, bestätigen die hohe Prävalenz von chronischen Venenkrankheiten [7]. Lediglich 9,6\% der Probanden hatten keinerlei pathologische Venenveränderungen.

geprägte Varizen ohne die typischen Zeichen einer CVI. Dagegen waren die fortgeschrittenen Stadien der CVI mit trophischen Störungen an der Haut bis hin zum Ulcus cruris mit 3,6\% deutlich niedriger als in der „Tübinger Studie“ (13\%). Der Anteil klinisch schwerer Stadien der Erkrankung mit typischen chronisch-venösen Hautveränderungen ist damit im Vergleich zu früheren Studien aus dem deutschen Sprachraum auffällig gering [7]. Das könnte dafür sprechen, dass bei gleich bleibend hoher Prävalenz von Venenerkrankungen eine höhere Therapierate vorliegt. Eine vergleichbare, relativ niedrige Rate klinisch schwerster Erscheinungsformen der chronischen venösen Insuffizienz zeigte sich auch in der „Edinburgh Vein Study“ sowie in den Ergebnissen des „San Valentino Screening Projekts“ [8-10].

\section{Primäre Varikosis \\ $\nabla$}

Unter dem Begriff der primären Varikosis werden erworbene oder angeborene Erweiterungen von Venen zusammengefasst, die einen suffizienten Klappenschluss unmöglich machen. Es kommt zur Schlängelung der Venen, die überdies eine unterschiedliche Wanddicke aufweisen können [11,12] ( Abb. 1).

Hämodynamisch relevant ist insbesondere die Stammvarikosis der Vv. saphena magna und parva. Sie wird nach Hach entsprechend der Refluxausdehnung von proximal nach distal in 4 bzw. 3 Stadien eingeteilt ( $\bullet$ Tab. 1).

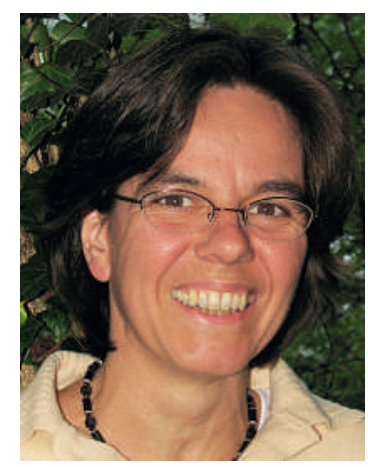

PD Dr. med. Birgit Kahle

Bibliografie

DOI $10.1055 / \mathrm{s}-0029-1214668$

Akt Dermatol 2009; 35 :

243-255 ๔ Georg Thieme

Verlag KG Stuttgart · New York ISSN 0340-2541

Korrespondenzadresse PD Dr. med. Birgit Kahle Klinik für Dermatologie, Allergologie und Venerologie Universitätsklinikum Schleswig-Holstein Campus Lübeck Ratzeburger Allee 160 23538 Lübeck

birgit.kahle@uk-sh.de 
Abb. 1 Primäre Varikosis.

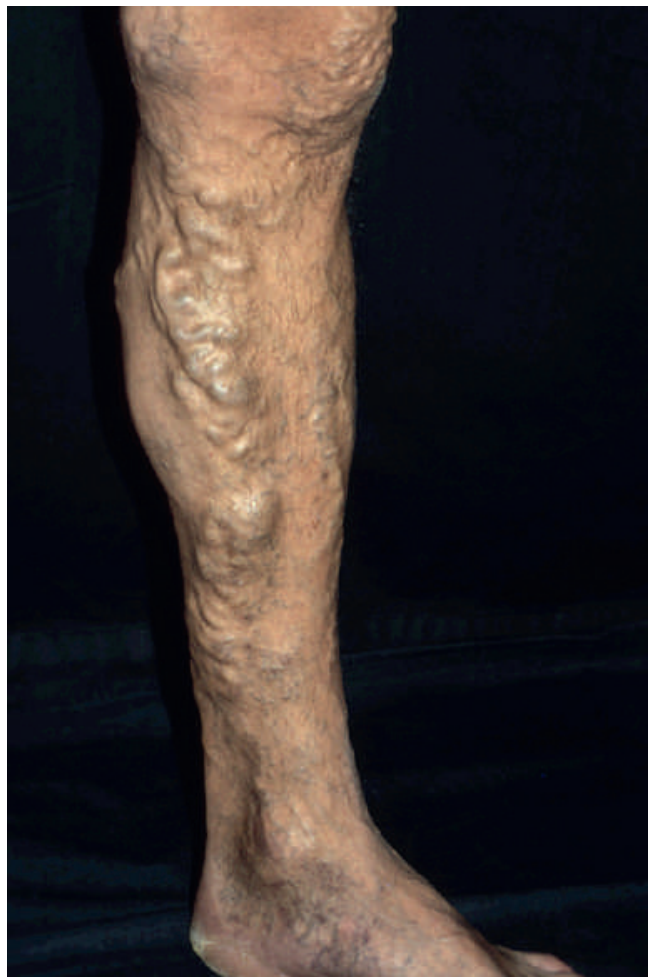

Tab. 1 Stadieneinteilung der Stammvarikosis (Refluxstrecke der Stammvenen) modifiziert nach Hach [13].

\begin{tabular}{|c|c|c|}
\hline Stadium & Vena saphena magna & Vena sephena parva \\
\hline I & Insuffizienz der Mündungsklappen & Insuffizienz der Mündungsklappen \\
\hline II & $\begin{array}{l}\text { Insuffizienz der Venenklappen } \\
\text { mit retrogradem Blutstrom bis } \\
\text { oberhalb des Knies }\end{array}$ & $\begin{array}{l}\text { Insuffizienz der Venenklappen } \\
\text { mit retrogradem Blutstrom bis } \\
\text { zur Wadenmitte }\end{array}$ \\
\hline III & $\begin{array}{l}\text { Insuffizienz der Venenklappen } \\
\text { mit retrogradem Blutstrom bis } \\
\text { unterhalb des Knies }\end{array}$ & $\begin{array}{l}\text { Insuffizienz der Venenklappen } \\
\text { mit retrogradem Blutstrom bis } \\
\text { zur Knöchelregion }\end{array}$ \\
\hline IV & $\begin{array}{l}\text { Insuffizienz der Venenklappen } \\
\text { mit retrogradem Blutstrom bis } \\
\text { zur Knöchelregion }\end{array}$ & \\
\hline
\end{tabular}

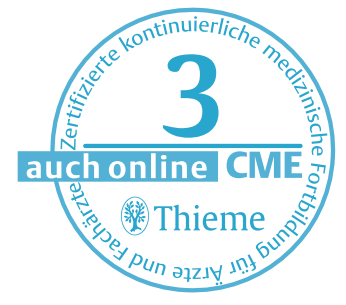

\section{Ätiologie}

Die genaue Ätiopathogenese der primären Varikosis ist bislang noch nicht geklärt. Diskutiert werden hämodynamische Aspekte als Folge primär insuffizienter Venenklappen. Als besonders bedeutsam wird diesbezüglich die Mündungsregion der V. saphena magna in die V. femoralis communis betrachtet $[14,15]$. Cotton vertrat erstmals die Auffassung, dass die Klappeninsuffizienz als Folge einer primären Dilatation (Degeneration) der Venenwand auftritt [16]. Die Wandschwäche von Venen als Ursache für die Ausbildung einer Klappeninsuffizienz und letztlich einer chronischen venösen Insuffizienz wurde von Thulesius eingehend untersucht [17]. Elektronenoptische Untersuchungen zeigen typische strukturelle Veränderungen der Myozyten der varikösen Venenwand sowie eine deutliche Erweiterung des intermyozytären Raumes, einhergehend mit Veränderungen der Interzellularsub- stanz, vornehmlich des Kollagens [18-21]. Es gibt Hinweise, dass die Veränderungen im Aufbau der Venenwand mit extrazellulären Matrixremodellierenden Proteinen (Metalloproteinasen MMP-1, -3 und -13) und Serinprotease (Tryptase) zusammenhängen [5]. Da viele Funktionen der Venenwand, wie die Kontraktion ihrer Myozyten, durch endotheliale Faktoren reguliert werden, kommt dem Endothel ebenfalls pathogenethische Bedeutung für die Varikosis zu.

Letztlich bleibt aber immer noch unklar, ob die endothelialen Faktoren, die ebenso wie lysosomale Enzyme im Serum von Varizenpatienten nachweisbar sind [22], primäre ätiologische Bedeutung besitzen oder ob sie nicht als Folge einer venösen Hypertension oder Stase mit resultierender Hypoxie sekundär auftreten [19]. Zusammenfassend kann gesagt werden, dass Veränderungen sowohl des Endothels als auch der Media (metabolische Affektion der Myozyten) mit einer unphysiologischen Druckerhöhung bei venöser Stase und Hypoxie in engem Zusammenhang stehen [18].

Für eine genetische Disposition der primären Varikosis spricht die auffällige familiäre Häufung venöser Erkrankungen, wie sie in zahlreichen epidemiologischen Studien und Untersuchungen gezeigt werden konnte $[4,23]$.

Die primäre Varikosis ist ein polyätiologisches Geschehen.

\section{Chronische venöse Insuffizienz}

$\nabla$

\section{Pathophysiologie}

Sowohl die primäre Varikosis mit dilatierten oberflächlichen oder tiefen Venen und insuffizienten Venenklappen als auch sekundäre Varizen bei postthrombotischem Syndrom können $\mathrm{zu}$ einer chronischen venösen Insuffizienz (CVI) führen. Wesentliche pathophysiologische Grundlage ist die ambulatorische venöse und kapilläre Hypertonie [24,25].

Als pathogenetisch bedeutsam für die CVI gilt die Insuffizienz der tiefen Leitvenen [26, 27] mit der damit in Zusammenhang stehenden Volumenüberlastung der tiefen Venen.

Trendelenburg prägte in diesem Zusammenhang den Begriff des Privatkreislaufs, der die Grundlage für die Untersuchungen über die pathophysiologische Bedeutung der Rezirkulationskreise der primären Varikosis von Hach bildete $[28,29]$. So fließt das Blut in insuffizienten epifaszialen (Stamm-)Venensegmenten vornehmlich in aufrechter Körperhaltung als auch bei intraabdomineller oder proximaler Druckerhöhung (Valsalva) 
retrograd. Über distale Perforansvenen (distaler Insuffizienzpunkt) führt der venöse Abstrom erneut in das tiefe Leitvenensystem und kann über den proximalen Insuffizienzpunkt (z.B insuffiziente Mündung der V. saphena magna in die V. femoralis) erneut zurückfließen. Infolge der anhaltenden Druck- und Volumenbelastung können im weiteren Verlauf der Erkrankung auch die distalen Perforansvene und die tiefen Leitvenen insuffizient werden.

Das Ulcus cruris venosum gilt als die schwerwiegendste Folgererkrankung der venösen Insuffizienz mit einer enormen sozioökonomischen Bedeutung. Aus diesem Grund ist eine frühzeitige diagnostische Abklärung und Therapie der Varikosis dringend zu empfehlen.

\section{Stadieneinteilung}

Viele Jahre war im deutschen Sprachraum die Klassifikation der CVI in 3 klinische Stadien in Anlehnung an die Einteilung von Widmer [6] gebräuchlich ( $\bullet$ Tab. 2).

Inzwischen ist die internationale CEAP-Klassifikation etabliert [30], die neben klinischen Symptomen der CVI ( $\odot$ Tab. 3) auch anatomische, ätiologische und pathophysiologische Aspekte berücksichtigt.

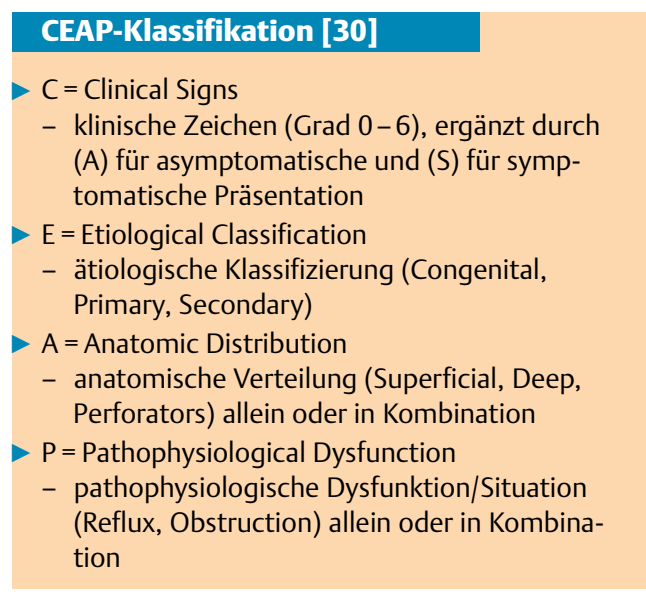

\section{Diagnostische Schritte zur Abklärung der Varikosis \\ $\nabla$}

\section{Varikosis-spezifische Anamnese}

Typische anamnestisch angegebene Beschwerden bei der Varikosis sind nächtliche Wadenkrämpfe, ein Schweregefühl der Beine sowie Schwellungsneigung der Beine zum Abend hin. Die Patienten geben in der Regel eine Besserung der Beschwerden bei Bewegung sowie bei Hochlagerung der Beine oder Betätigung der Wadenmuskelpumpe an.
Tab. 2 Stadieneinteilung der CVI modifiziert nach Widmer [6].

\begin{tabular}{|l|l|}
\hline Stadium & Merkmale \\
\hline Stadium I & Corona phlebectatica paraplantaris, Phlebödem bzw. Knöchelödem \\
\hline Stadium II & zusätzlich trophische Störungen mit Ausnahme des Ulcus cruris \\
\hline Stadium III & Ulcus cruris ( IIla abgeheiltes Ulkus, IIIb florides Ulkus) \\
\hline
\end{tabular}

Tab. 3 Klinischer Score C0 - C6 der CEAP-Klassifikation.

\begin{tabular}{ll} 
Score & Merkmale \\
\hline$C_{0}$ & Keine sichtbaren oder tastbaren Zeichen einer venösen Erkrankung \\
\hline$C_{1}$ & Teleangiektasien oder retikuläre Varizen \\
\hline$C_{2}$ & Varizen \\
\hline$C_{3}$ & Phlebödem \\
\hline$C_{4 a}$ & Hautveränderungen (z. B. Ekzem, Hyperpigmentierungen, Liposklerose) \\
\hline$C_{4 b}$ & Lipodermatosklerose oder Atrophie blanche \\
\hline$C_{5}$ & Hautveränderungen wie bei $C_{4}$ mit abgeheiltem Ulkus \\
\hline$C_{6}$ & Hautveränderungen wie oben mit floridem Ulkus \\
\hline
\end{tabular}

\section{Klinische Untersuchung}

Inspektorisch fallen bei der Varikosis Teleangiektasien am medialen Fußrücken, Hyperpigmentierungen am Unterschenkel, ggf. auch Unterschenkelekzem, eine Atrophie blanche, Ulzerationen oder Narben nach Ulzerationen auf. Das typische Ulcus cruris venosum findet sich am medialen Knöchel zwischen Innenknöchel und Achillessehne.

\section{Apparative Diagnostik}

Ziel der phlebologischen Diagnostik ist eine klare, möglichst quantitative und qualitative Erfassung des jeweiligen venösen Krankheitsbildes. Für die Planung des therapeutischen Vorgehens ist in erster Linie eine bildgebende Diagnostik zu fordern.

In der diagnostischen Abklärung venöser Krankheitsbilder spielen derzeit nicht invasive funktionelle und bildgebende Verfahren die Hauptrolle.

Invasive diagnostische Methoden wie die direkte Messung des Venendrucks vor, während und nach Belastung oder die Phlebografie bleiben speziellen Fragestellungen vorbehalten.

\section{Digitale Photoplethysmografie}

Die Digitale Photoplethysmografie (DPPG) ist eine häufig angewandte, nicht invasive Screeningmethode in der venösen Funktionsdiagnostik, die auf den unterschiedlichen Reflexionseigenschaften der Haut entsprechend ihrem Durchblutungsgrad (Blutfülle) beruht [31 - 35]. Langwelliges Licht mit einem Absorptionsmaximum im nahen infraroten Bereich wird in die Haut eingestrahlt und der reflektierte Anteil registriert und aufgezeichnet. Die jeweilige Reflexion des Lichts wird im Wesentlichen durch den Füllungsgrad der in 2-3 mm Tiefe verlaufenden venösen Gefäßplexus bestimmt, der sich wäh-

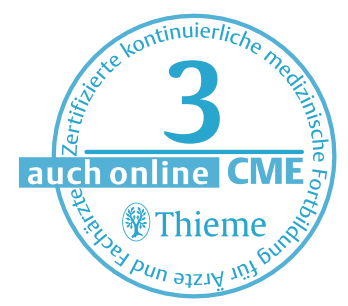




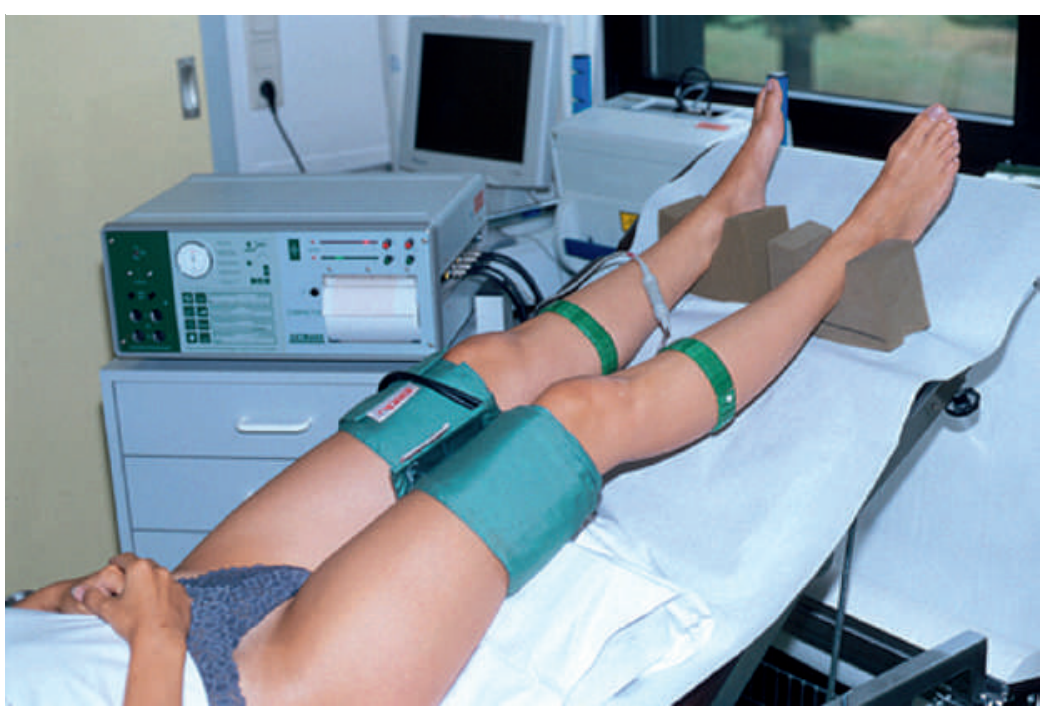

Abb.2 Venenverschlussplethysmografie.

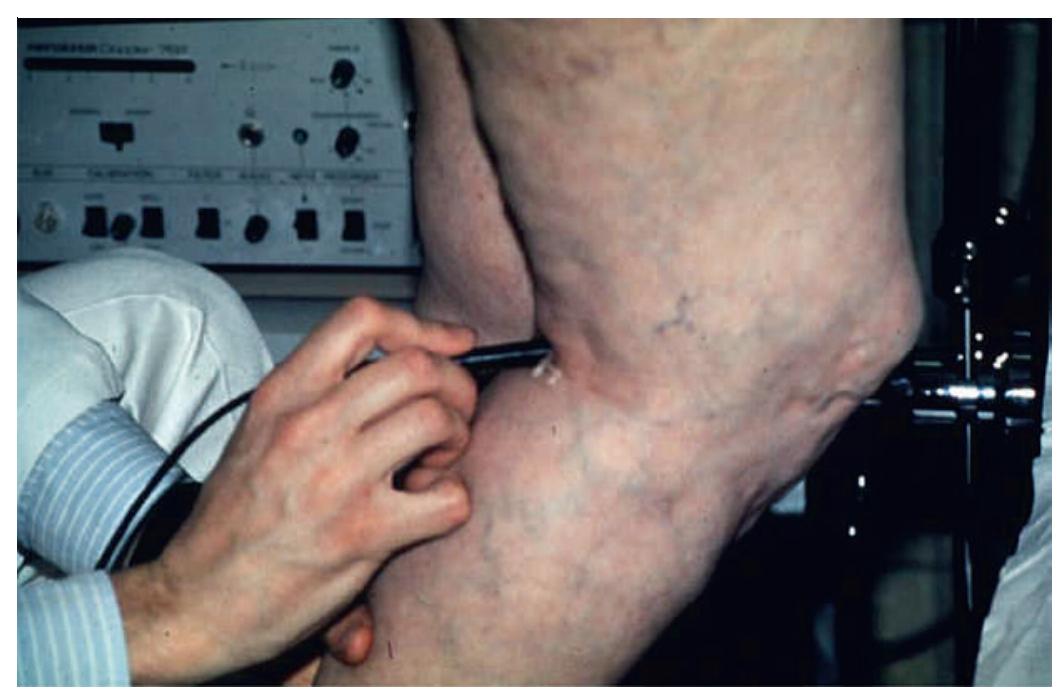

Abb. 3 Dopplersonografie der V. saphena parva.

rend eines definierten Bewegungsprogramms ändert $[36,37]$. Bei der DPPG werden die Änderungen der venösen Blutfülle und die damit zusammenhängenden Reflexionseigenschaften im Vergleich zu einem Ausgangswert erfasst. Als Hauptparameter wird die Zeit bis zum Wiedererreichen des Ausgangsniveaus (Wiederauffüllzeit t0) in Sekunden gemessen. Durch Anlegen eines Tourniquets erlaubt die DPPG auch Rückschlüsse über die venöse Abstromleistung der tiefen Beinvenen.

\section{Venenverschlussplethysmografie}

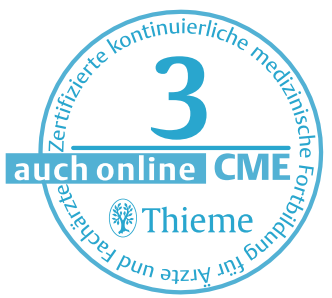

Die Venenverschlussplethysmografie (VVP) erlaubt wie die DPPG globale Aussagen über die Venenfunktion eines Beines. Nach Anlage eines supradiastolischen Staudrucks am Oberschenkel werden in definierter Position über Dehnungsmessstreifen an der Wade Aussagen über die venöse Kapazität (ml/100 ml Gewebe) und über den venösen Ausstrom (ml/100 ml Gewebe/min) nach Ablassen des Staus getroffen ( $\bullet$ Abb. 2).

\section{Direktionale Dopplersonografie}

Auch wenn die Duplexsonografie aus der phlebologischen Routinediagnostik nicht wegzudenken ist, hat die uni- und bidirektionale Dopplersonografie für die Fragestellung „refluxive Venenerkrankung? Ja oder nein?" noch eine gewisse Bedeutung ( $\bullet$ Abb.3). Anatomische Aussagen lassen sich allerdings nur sehr eingeschränkt vornehmen. Für die Messung des systolischen Blutdrucks der Unterarme und Knöchel, also zur Bestimmung des Knöchel-Arm-Index ist die Dopplersonografie nach wie vor die Methode der Wahl.

\section{Duplexsonografie}

Als nicht invasive bildgebende Untersuchungsmethode besitzt die Duplexsonografie insbesondere in der prätherapeutischen Diagnostik eine herausragende Stellung. Mittels Duplex lassen sich hämodynamische Werte quantitativ erfassen. Refluxstrecken, anatomische Besonderheiten und der Bezug der Varizen zu Nachbarstrukturen lassen sich reproduzierbar dokumentieren. Zur Dokumentation von Refluxen bei Varikosis ist ein Ausdruck oder die digitale Speicherung eines simultan zur B-Bildsonografie abgeleiteten pw-Dopplersignals zu fordern.

Die Duplexsonografie dient nicht nur der Diagnosestellung, sondern sie stellt vielmehr eine wichtige Hilfe bei der Planung des im individuellen Falle geeigneten therapeutischen Vorgehens dar.

Hinsichtlich einer quantitativen Evaluierung der venösen Hämodynamik besitzt die Duplex-Sonografie einen besonderen Stellenwert. Sie ermöglicht neben einer exakten morphologischen Diagnostik die gezielte Untersuchung von Fließeigenschaften des Blutes sowie von pathologischen Refluxen in einzelnen Gefäßabschnitten des Venensystems ( Abb. 4 und 5) [38-45]. Bestimmte Parameter in der duplexsonografischen Refluxdiagnostik wie Refluxvolumen, Refluxgeschwindigkeit oder Refluxdauer eignen sich zur hämodynamischen Abklärung bei Varikosis, allerdings lassen sich derartige Refluxmessungen oder eine vergleichbare Refluxprovokation nur mit großem Aufwand standardisieren [46-49].

\section{Therapie}

$\nabla$

Die Indikation zur Therapie der Varikosis orientiert sich an den anatomischen und pathophysiologischen Gegebenheiten sowie am Beschwerdebild des Patienten. 
Ziel der Therapie sind die Normalisierung oder Besserung der venösen Hämodynamik und insbesondere die Vermeidung von Folgeschäden durch die Varikosis (z. B. Stauungsekzeme, Ulcus cruris venosum, trophische Störungen, arthrogenes Stauungssyndrom, Varikophlebitis).

Die Therapie der Varikosis fußt dabei im Wesentlichen auf 6 Säulen:

- konservative Maßnahmen

- Sklerosierungstherapie

- endoluminale thermische Verfahren

- transkutane Lasertherapie

- operative Verfahren

- medikamentöse Therapie

Der jeweilige Therapieplan sollte individuell zusammengestellt werden und hängt ab vom Allgemeinzustand des Betroffenen, den geschädigten Venenabschnitten (z.B. Stammvarize vs. Seitenastvarize), dem Durchmesser und Verlauf der geschädigten Vene sowie den bereits erfolgten Vortherapien. In der Regel ist eine Kombination verschiedener Maßnahmen sinnvoll [50].

\section{Konservative Therapie}

Die konservative Therapie umfasst unterschiedliche Arten von entstauenden und komprimierenden Maßnahmen, die in allen Stadien der Varikosis allein oder in Kombination mit interventionellen oder operativen Verfahren eingesetzt werden können [51]. Hierzu gehören: phlebologische Kompressionsverbände, medizinische Kompressionsstrümpfe, manuelle Lymphdrainage, apparative intermittierende Kompression und der Gefäßsport.

Bei der konservativen Therapie sollte insbesondere auf eine Betätigung der Wadenmuskelpumpe sowie auf eine ausreichende Beweglichkeit im Sprunggelenk geachtet werden.

Eine alleinige Einschränkung der Beweglichkeit im Sprunggelenk kann bereits zu Stauungsbeschwerden führen (arthrogenes Stauungssyndrom). Bei älteren, multimorbiden Personen können der Kompressionstherapie Grenzen gesetzt sein durch z. B. kardiale Insuffizienz, fortgeschrittene periphere arterielle Verschlusskrankheit (pAVK) oder Neuropathie. Eine unsachgemäße Anwendung von Kompressionsmaßnahmen kann Spannungsblasen, Hautnekrosen und nervale Druckschäden zur Folge haben.

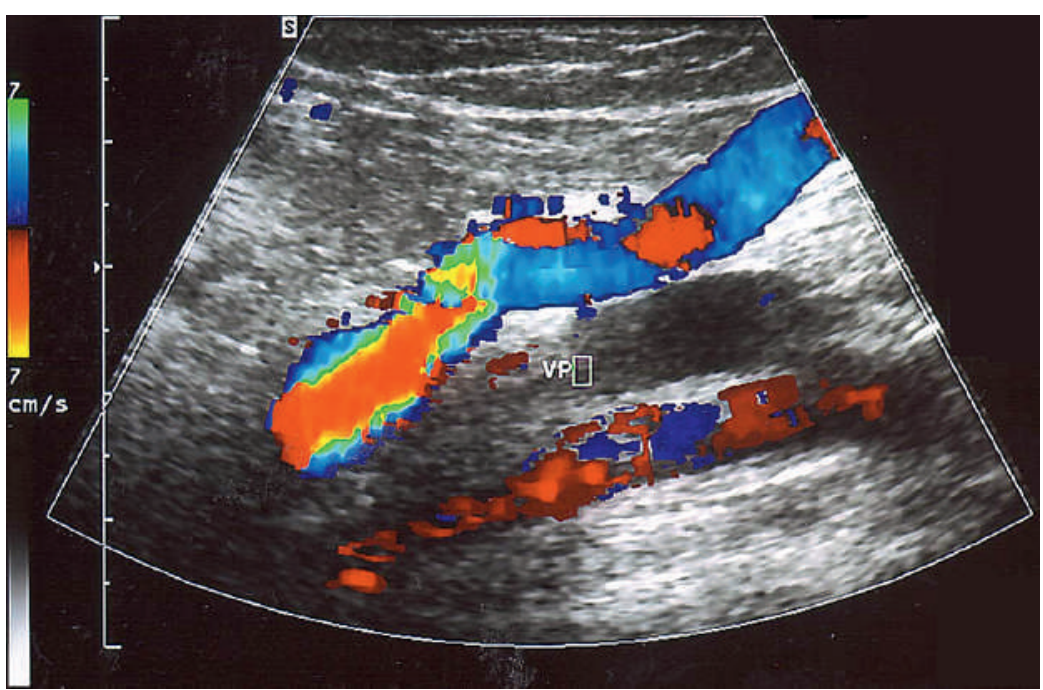

Abb. 4 Duplexsonografie der V. saphena parva.

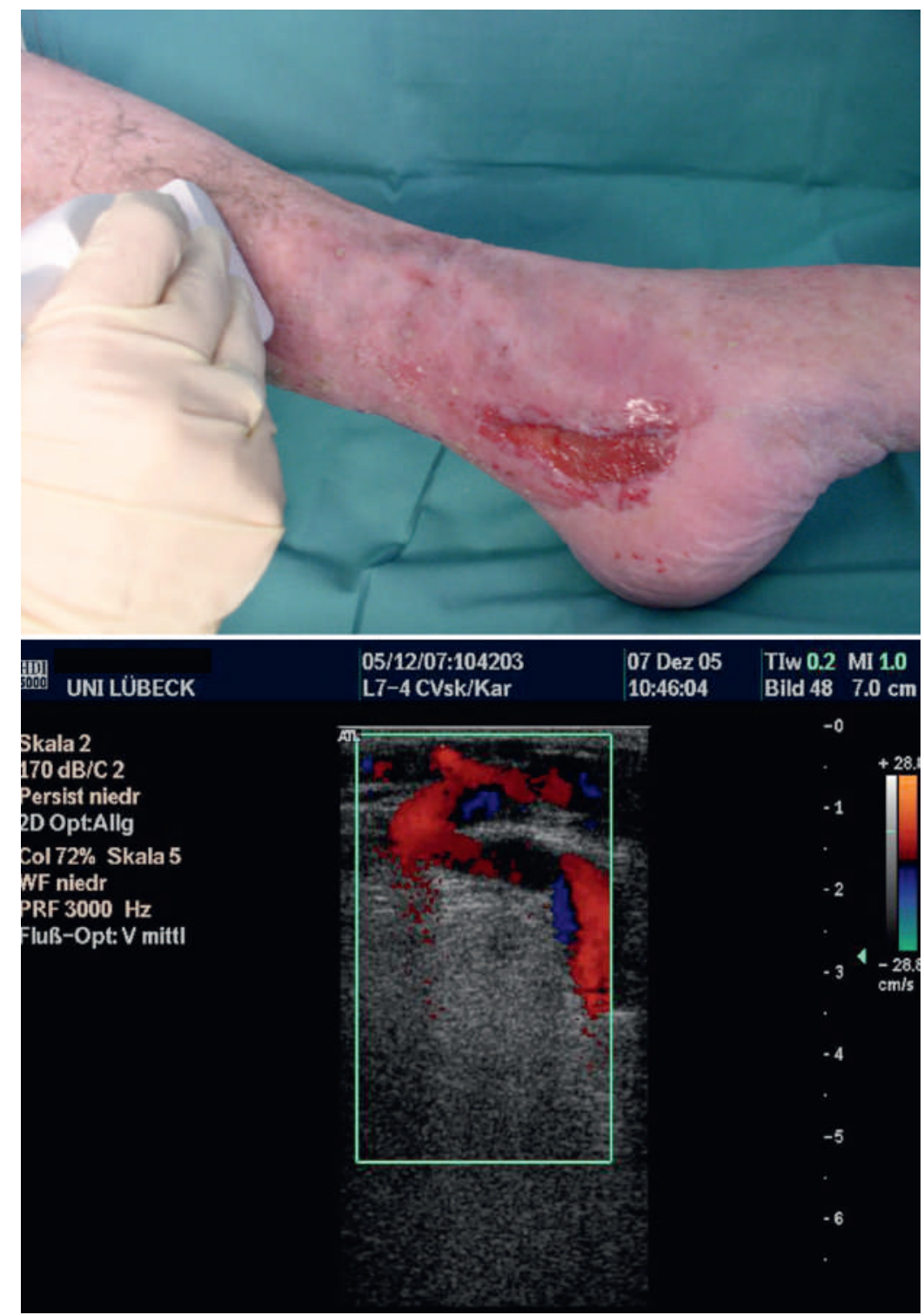

Abb. 5 Duplexsonografie von insuffizienten Perforansvarizen, pathologischer Reflux. 


\section{Kompressionstherapie}

Die Kompressionstherapie gilt nach wie vor als Basistherapie für venöse Erkrankungen.

Neben phlebologischen Kompressionsverbänden stehen Kompressionsstrümpfe in unterschiedlichen Kompressionsklassen und Größen zur Verfügung.

Die Wirkungsweise der Kompressionstherapie auf die venöse Situation ist komplex. So führt das Tragen von Kompressionsstrümpfen bei Patienten mit einer venösen Insuffizienz zu einer nachweisbaren Verbesserung der Hämodynamik [52 -57]. Kompressionsverbände unterschiedlicher Elastizität weisen unterschiedliche Eigenschaften auf die venöse Hämodynamik auf, insbesondere erweisen sich unelastische Materialien als effektiver hinsichtlich einer Refluxbehandlung als elastische Kompressionsverbände [58]. Aber auch Kompressionsstrümpfe derselben Kompressionsklasse besitzen unterschiedliche Akuteffekte auf die venöse Hämodynamik [59]. Die Wirksamkeit der verschiedenen Kompressionsartikel liegt in erster Linie an der Dehnbarkeit des Materials. In vivo lässt sich dies durch das Verhältnis von maximalem Anpressdruck bei Bewegung zu dem Ruhedruck im Stehen charakterisieren.

Ferner hat die Kompressionstherapie einen großen Einfluss auf die Mikrozirkulation im Hautorgan [24]. Die herausragende Bedeutung der Kompressionstherapie in der Behandlung venöser Ulzera ist durch zahlreiche Studien und Metaanalysen belegt (Cochrane). Gleiches gilt für die nachweisbare Effektivität der Kompression in der Ödemreduktion. In vitro lassen sich die Einflüsse von Kompression auf Rezeptorebene nachweisen, was hilft, den klinisch fassbaren Effekt besser zu verstehen [60]

\section{Medikamentöse Therapie}

Systemisch wirksame Venentherapeutika werden vor allem bei Schwellneigung und Schweregefühl im Rahmen einer venösen Insuffizienz eingesetzt. Ein eindeutig positiver Effekt auf die Ödementwicklung konnte unter anderem bei rotem Weinlaubextrakt, Rosskastanienextrakt, Oxerutin und Calciumdobesilat nachgewiesen werden [61]. Es muss betont werden, dass diese systemischen adjuvanten Therapien kein dauerhafter Ersatz für eine kausale Therapie durch eine Sanierung einer Varikosis oder für eine Kompressionstherapie sein können. Die möglichen Nebenwirkungen und die damit verbundenen Kontraindikationen sind eher als gering einzuschätzen $[62,62]$.
Interventionelle Therapien

Sklerosierungstherapie

Ziel der Verödungsbehandlung ist, durch Injektion einer gewebetoxischen Flüssigkeit (einiziges in der BRD zugelassenes Sklerosierungsmittel ist derzeit Aethoxysklerol ${ }^{\circledR}$, Wirkstoff: Polidocanol) in eine Varize einen lokalen Endothelschaden zu erzeugen, der zu einer Obliteration und Fibrosierung des Gefäßes führt.

Die wesentliche Bedeutung der Sklerosierung liegt in der Behandlung von Seitenastvarizen sowie Besenreiser- und retikulären Varizen.

Auch Perforans- und Stammvenen sind einer Verödungstherapie prinzipiell zugänglich, sollten allerdings nicht mittels flüssiger sondern aufgeschäumter Sklerosierungsmittel therapiert werden. Für die duplexkontrollierte Schaumsklerosierungstherapie konnte im Vergleich zur Flüssigsklerosierung eine deutlich verbesserte Effektivität nachgewiesen werden [64].

Unter Beachtung der Indikationen und (relativen) Kontraindikationen stellt die Verödungstherapie ein effektives, kostengünstiges und komplikationsarmes Behandlungsverfahren der Varikosis dar ( Tab.4). Als häufigere Nebenwirkungen sind Thrombophlebitiden, allergische Reaktionen, Flimmerskotome, Pigmentierungsstörungen, Matting und sehr selten thromboembolische Komplikationen zu nennen [65]. Insbesondere ältere, multimorbide Patienten, denen eine Varizenoperation nicht zuzumuten ist, profitieren von einer Sklerosierungstherapie. Auch für Patienten mit einer fortgeschrittenen Varikosis und einem Ulcus cruris venosum stellt die Schaumsklerosierungstherapie periulzeröser Varizen eine effektive Theapieoption dar [66]. Sogar Patienten mit einem postthrombotischen Syndrom oder unter laufender oraler Antikoagulation profitieren von der Therapie [67]. Es kommt zu einer rascheren Abheilung des Ulcus cruris venosum im Vergleich zur alleinigen Kompressionstherapie.

Eine Weiterentwicklung der Schaumsklerosierungstherapie mittels Direktpunktion der Vene stellt die Katheter-assistierte Sklerosierungstherapie der V. saphena magna dar. Hier sind bisher 2 verschiedene Kathetertypen im Einsatz. Wildenhues verwendete einen einlumigen Angiografiekatheter zum Einbringen des Sklerosierungsmittels in die Zielvene [68]. Brodersen et al. nutzten einen doppellumigen Ballonkatheter. Der Ballon erlaubt das Abdichten der Vene zur Krosse, was den Übertritt von Sklerosierungsschaum in das tiefe Venensystem mindern soll [69].

Einen besonderen Stellenwert besitzt die Schaumsklerosierungstherapie in der Behandlung von Rezidivvarizen. So können insbesondere stark geschlängelte dünnwandige und oft kleinlu- 
mige Rezidivvarizen in den Krossen effektiv und minimal invasiv behandelt werden ( $\bullet$ Abb. 6).

\section{Transkutane Lasertherapie}

Die Transkutane Lasertherapie konkurriert mit der Flüssigsklerosierung in der Therapie von Besenreisern und retikulären Varizen. Hierbei werden von außen Besenreiservarizen mittels verschiedener Lasertypen koaguliert. Die mäßigen Erfolgsraten der Lasertherapie von Besenreisern und retikulären Varizen, die Nebenwirkungen und die vergleichsweise hohen Kosten sprechen nach dem derzeitigen Stand der Literatur eher für die herkömmliche Sklerosierungstherapie.

\section{Operative Therapie}

Unabdingbar für das operative Vorgehen bei Varikosis ist eine exakte präoperative Diagnostik zur Erfassung der individuellen anatomischen und hämodynamischen Gegebenheiten.

Die Ziele der operativen Verfahren sind die dauerhafte Entfernung von Varizen unter Berücksichtigung der Invasivität des Eingriffs und des kosmetischen Ergebnisses. Allgemein lassen sich die Prinzipien der modernen Varizenchirurgie knapp definieren: Es gilt, den proximalen und den distalen Insuffizienzpunkt und die dazwischen liegenden varikösen Venenabschnitte auszuschalten.

\section{Krossektomie und Stripping}

Unter Venenoperationen werden in der Regel die klassische Krossektomie und Strippingoperation der Stammvenen in Verbindung mit der Miniphlebektomie von Seitenästen verstanden. Das klassische Stripping geht auf W. Babcock zurück, der 1907 eine neue Methode zur Entfernung von Varizen veröffentlichte [70]. Ein Stripping der V. saphena magna wird derzeit im Gegensatz zu der von Babcock beschriebenen Verfahrensweise von der Leiste bis unterhalb des Knies durchgeführt.

Im Unterschenkelbereich kann es beim Strippen zu Schädigungen des N. saphenus kommen (in bis zu $30 \%$ der Fälle).

Dieses Risiko ist bei der Miniphlebektomie mit Häkchen, bei der die Saphenaloge am Unterschenkel nicht tangiert wird, deutlich geringer. In vielen Fällen ist bei einer Saphena-magna-Insuffizienz der Saphena-Hauptstamm am Unterschenkel kompetent und die hintere und vordere Bogenvene dagegen varikös degeneriert. Von daher macht in diesen Fällen die Kombination aus Teilstripping der V. saphena magna mit selektiver Exhairese der Seitenäste Sinn.
Tab. 4 Kontraindikationen der Sklerosierungstherapie.

\begin{tabular}{ll} 
Absolute Kontraindikationen der & Relative Kontraindikationen der \\
Sklerosierungstherapie & Sklerosierungstherapie \\
- bekannte Allergie auf das & - Beinödem, nicht kompensiert \\
Sklerosierungsmittel & - diabetische Spätkomplikationen \\
- schwere Systemerkrankung & (z. B. Polyneuropathie) \\
- akute tiefe Beinvenenthrombose & - arterielle Verschlusskrankheit im \\
- lokale, im Bereich der Sklerosierung & Stadium II \\
gelegene oder schwere generalisierte & - schlechter Allgemeinzustand \\
Infektionen & - Bronchialasthma \\
- längerfristige Immobilität oder & - ausgeprägte allergische Diathese \\
Bettlägerigkeit & - bekannte Thrombophilie oder \\
- fortgeschrittene arterielle Verschluss- & Hyperkoagulabilität mit oder ohne \\
krankheit im Stadium III oder IV & abgelaufene tiefe Beinvenenthrombose \\
- Hyperthyreose (bei jodhaltigen & - für die Schaumsklerosierung: \\
Sklerosierungsmitteln) & bekanntes asymptomatisches offenes \\
- Schwangerschaft (außer bei & Foramen ovale \\
zwingender Indikation) & hohes Risiko für thrombembolische \\
- für die Schaumsklerosierung: & Ereignisse \\
bekanntes symptomatisches offenes & Sehstörungen oder neurologische \\
Foramen ovale & Störungen nach vorangegangener \\
& Schaumsklerosierung \\
\hline
\end{tabular}

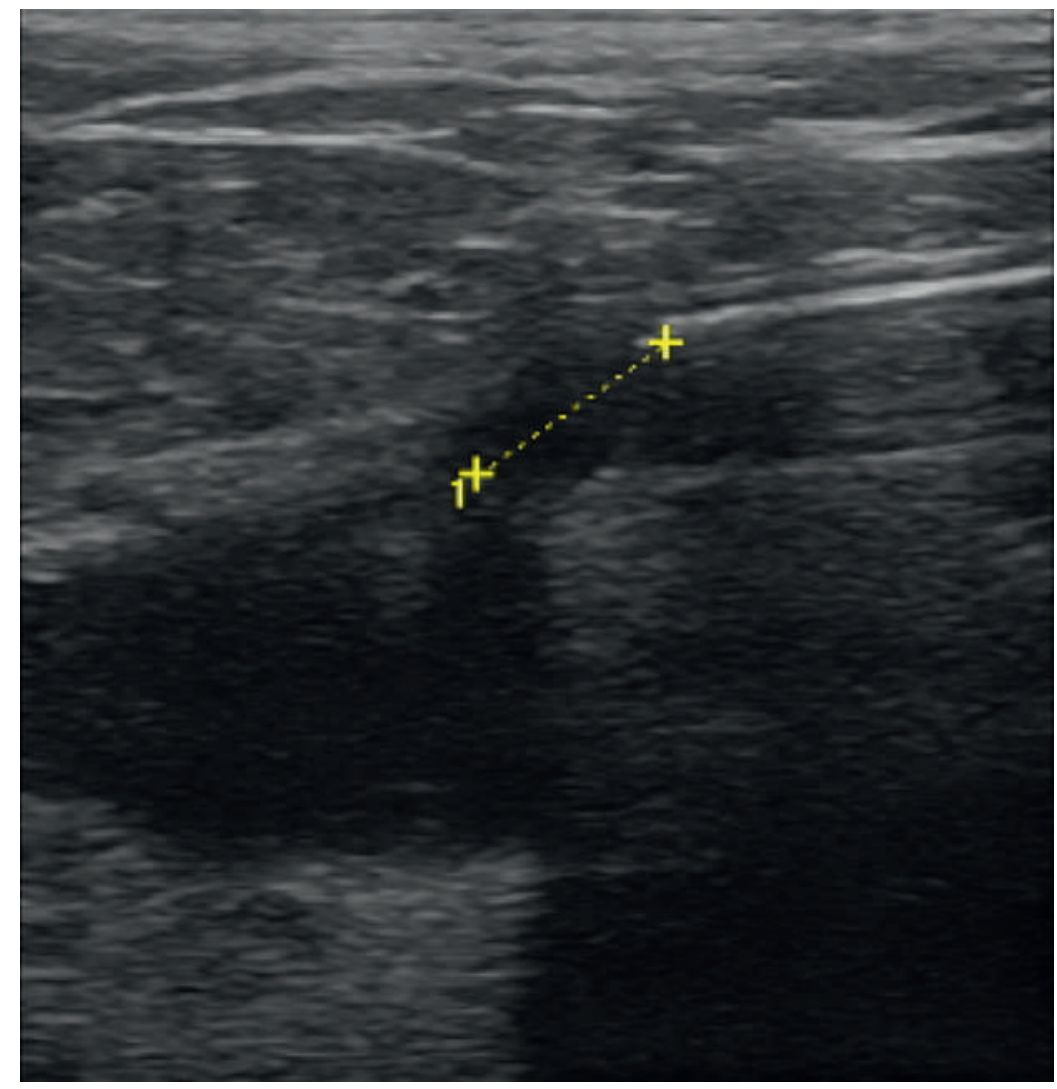

Abb. 6 Platzieren des Closure-

Nach Hach stellt die partielle Resektion der V. saFast-Applikators in der Leiste. phena magna in solchen Fällen die logische bzw. therapeutische Konsequenz der Kenntnis des Operateurs von der Bedeutung der pathologischen Rezirkulationskreise dar [28,29].

Es existieren verschiedene Techniken für das Entfernen der Stammvenen mittels eines Strippers. Die Invaginationstechnik zeichnet sich durch eine Schonung des Umgebungsgewebes, eine geringe Rate von Nachblutungen sowie eine geringere postoperative Schmerzhaftigkeit aus $[71,72]$. Zur 
Entfernung der V. saphena parva wie auch der V. saphena magna eignet sich der PIN-Stripper nach Oesch, wodurch die Größe des Schnittes am distalen Insuffizienzpunkt minimiert werden kann [73]. Beim Kryostripping kann auf den Schnitt am distalen Insuffizienzpunkt vollständig verzichtet werden [74]. Es eignet sich wegen der starren Kryosonde, die von der Leiste her in die V. saphena magna eingeführt wird, nur eingeschränkt für stärker geschlängelte Varizen.

Die CHIVA-Methode (Cure conservatrice et hémodynamique de l'insuffisance veineuse en ambulatoire) beinhaltet die gezielte Unterbrechung der Rezirkulationskreisläufe bzw. die gezielte Ausschaltung der Insuffizienzpunkte unter Belassen der Stammvenensegmente [75]. Das Verfahren ist wissenschaftlich noch nicht abschließend beurteilbar und wird eher kritisch gesehen.

Die subfasziale endoskopische Perforansdissektion (SEPS) spielt derzeit eine nur noch untergeordnete Rolle bei venenchirurgischen Eingriffen. Eine gezielte isolierte Ausschaltung insuffizienter Perforansvenen am Unterschenkel kann z.B. auch mit sonografisch kontrollierter Schaumsklerosierung erreicht werden.

\section{Endoluminale thermische Verfahren}

Neben der oben genannten kathetergestützen Sklerosierungstherapie ist die endoluminale Behandlung der Stamm-Varikosis mit Hilfe einer Laser- oder Radiowellentherapie (in Tumeneszenzanästhesie) möglich. Bei der endoluminalen Lasertherapie kommt es durch eine Gewebeerhitzung zu einer Kollagendenaturierung in der Venenwand. Auch bei der Radiowellentherapie ist das Therapieziel eine Schädigung der Venenwand mit einer konsekutiven Schrumpfung und Thromboisierung des Gefäßes. Beide Verfahren werden in der Regel in Tumeszenz-Lokalanästhesie durchgeführt, die nach sonografisch kontrollierter Platzierung des jeweiligen Kathetersystems paravasal in die Faszienloge der V. saphena magna oder V. saphena parva appliziert wird. Die Injektion der Tumeszenzlösung erfolgt ebenfalls sonografisch kontrolliert. Sie dient neben der Schmerzausschaltung der Vermeidung von Hitzeschäden umgebender Gewebe, insbesondere der darüber liegenden Haut.

Die Datenlage zur endoluminalen Lasertherapie ist schwer zu übersehen, da verschiedene Lasersysteme mit unterschiedlichen Wellenlängen und uneinheitlicher Energiedichte bzw. Fluence zum Einsatz kommen. Proebstle konnte zeigen, dass der nachhaltige Erfolg einer endovenösen Lasertherapie Fluence-abhängig ist [76]. Insgesamt sind die Ergebnisse nach endovenöser Lasertherapie positiv zu bewerten.

Im Gegensatz dazu ist das Verfahren der Radiofrequenzobliteration übersichtlicher, da es weniger verschiedene Gerätesysteme gibt (VNUS Closure, VNUS Closure Fast, Celon bipolare Radiofre- quenzobliteration RIFFT). Darüber hinaus werden in prospektiven randomisierten Studien im Vergleich zur klassischen Strippingoperation bei allerdings kleiner Fallzall vergleichbar gute mittelfristige Ergebnisse gezeigt [77,78].

Relativ neu ist die extraluminale Valvuloplastie, ein Verfahren zur Wiederherstellung der Funktion der Schleusenklappe im Bereich der Krosse der V. saphena magna. Dabei macht man sich zunutze, dass die Klappeninsuffizienz der Schleusenklappe oft nur durch eine Lumenerweiterung der Vena bei intakten Klappensegeln ausgelöst wird. Wird nun das Lumen durch eine DacronUmmantelung der Vene ausreichend eingeengt, ist eine Wiederherstellung der normalen Klappenfunktion möglich. Das Verfahren ist besonders bei Patienten mit einer Stammvarikosis der V. saphena magna Grad I in Betracht zu ziehen [79]

\section{Zusammenfassung}

Für die Behandlung der Varikosis stehen unterschiedliche Behandlungsmöglichkeiten zur Verfügung, die nach umfassender Diagnostik individuell auf das jeweilige Krankheitsbild abgestimmt werden können. Insbesondere haben neue technische Entwicklungen wie die Duplexsonografie als nicht invasives bildgebendes Verfahren viel zum Verständnis der venösen Anatomie und Pathophysiologie beigetragen. Neue operative Verfahren sind bei vergleichbarer Effektivität weitaus weniger invasiv als die klassischen Methoden und werden darüber hinaus unter sonografischer Kontrolle durchgeführt.

\section{Literatur}

1 Vorstand der Dt. Ges. f. Gefäßchirurgie (Hrsg). Leitlinie zu Diagnostik und Therapie in der Gefäßchirurgie. Köln: Deutscher Ärzteverlag, 1998: $105 \mathrm{ff}$

2 Brand FN, Dannenberg AL, Abbott RD et al. The epidemiology of varicose veins: The Framingham Study. Am J Prev Med 1988; 4: 96-101

3 Eberth-Willershausen W, Marshall M. Prävalenz, Risikofaktoren und Komplikationen peripherer Venenerkrankungen in der Münchner Bevölkerung. Hautarzt 1984; 35 : $68-77$

4 Fischer $\mathrm{H}$, Hrsg. Venenleiden - Eine repräsentative Untersuchung in der Bundesrepublik Deutschland (Tübinger Studie). München: Urban und Schwarzenberg, 1981

5 Gillespie DL, Patel A, Fileta A et al. Varicose veins possess greater quantities of MMP-1 than normal veins and demonstrate regional variation in MMP-1 and MMP-13. J Surg Res 2002; 106: $233-238$

6 Widmer LK, Stählin HB, Nissen C et al. (Hrsg). Venen-, Arterien-Krankheiten, koronare Herzkrankheit bei Berufstätigen, Prospektiv-epidemiologische Untersuchung. Basler Studie I-III 1959-1978. Bern, Stuttgart, Wien: Hans Huber, 1981

7 Rabe E, Pannier-Fischer F, Bromen K et al. Bonner Venenstudie der Deutschen Gesellschaft für Phlebologie. Epidemiologische Untersuchung zur Frage der Häufigkeit und Ausprägung von chronischen Venenkrankheiten in der städtischen und ländlichen Wohnbevölkerung. Phlebologie 2003; 32: 1- 14 
8 Bradbury A, Evans C, Allan Pet al. What are the symptoms of varicose veins? Edinburgh Vein Study cross sectional population survey. Brit Med J 1999; 318: $353-356$

9 Cesarone MR, Belcaro G, Nicolaides AN et al. „Real“ epidemiology of varicose veins and chronic venous diseases: the San Valentino screening project. Angiology 2002; 53: 19-130

10 Ruckley CV, Evans C], Allan PL et al. Chronic venous insufficiency: Clinical and duplex correlations. The Edinburgh Vein Study of venous disorders in the general population. J VAsc Surg 2002; 36: 520-525

11 Charles AK, Gresham GA. Histopathological changes in venous grafts and in varicose and non-varicose veins. J Clin Pathol 1993; 46: 603-606

12 Porto LC, da Silveira PR, de Carvalho IJ et al. Connective tissue accumulation in the muscle layer in normal and varicose saphenous veins. Angiology 1995; 46: 243-249

13 Hach W. Phlebographische Untersuchungen zum Spontanverlauf der primären Varikosis. Phlebol Proktol 1980; 9: 62-165

14 Schultz-Ehrenburg U, Weindorf N, Matthes U et al. Prospektive epidemiologische Studie über die Entstehung der Krampfadern bei Kindern und Jugendlichen. Phlebol Proktol 1989; 18: 3-11

15 Rabe P, Rabe E. Zur Häufigkeit der Krosseninsuffizienz bei der genuinen Varicose. Phlebol Proktol 1989; 18: 267-269

16 Cotton LT. Varicose veins. Cross anatomy and development. Br ] Surg 1961; 48: 589-597

17 Thulesius O. Pathophysiologie der venösen Insuffizienz. In: Fischer $\mathrm{H}$ (Hrsg). Chronische Veneninsuffizienz - Pathogenese und medikamentöse Therapie. Ergebnisse der Angiologie Bd 30. Stuttgart: Schattauer, 1984: $9-13$

18 Staubesand J. Matrix-Vesikel und Mediadysplasie: ein neues Konzept zur formalen Pathogenese der Varikosis. Phlebol Proktol 1987; 17: 109- 140

19 Michiels C, Bouaziz N, Remacle J. Role of the endothelium and blood stasis in the appearance of varicose veins. Int Angiol 2002; 21: 1-8

20 Gandhi RH, Irizarry E, Nackmann GB et al. Analysis of the connective tissue matrix and proteolytic activity of primary varicose veins. J Vasc Surg 1993; 18: $814-820$

21 Travers JP, Brookes CE, Evans J et al. Assessment of wall structure and composition of varicose veins with reference to collagen, elastin and smoth muscle content. Eur J Vasc Endovasc Surg 1996; 11: 230-237

22 Niebes $P$, Laszt $L$. Recherche sur l'activiée des enzymes dans le metabolisme de mucoploysaccharides de veins saphènes sains et variqueuses. Angiologica 1971; 8: 7-16

23 Cornu-Thénard A, Boivin P, Baud JM et al. Importance of the familial factor in varicose disease. Clinical study of 134 families. J Dermatol Surg Oncol 1994; 20: $318-326$

24 Jünger M, Hahn U, Bort S et al. Bedeutung der kutanen Mikroangiopathie für die Entstehung von Stauungsdermatosen bei chronischer venöser Insuffizienz (CVI). Wien Med Wschr 1994; 144: 206 - 210

25 Partsch H. Zur Pathogenese des venösen Ulcus cruris. Hautarzt 1985; 36: $196-202$

26 Hach $W$, Schirmers $U$, Becker L. Veränderungen der tiefen Leitvenen bei einer Stammvarikosis der V. saphena magna. In: Müller-Wiefel H (Hrsg). Mikrozirkulation und Blutrheologie. Baden-Baden, Köln, New York: Witzstrock, 1980: $468-470$

27 Hach-Wunderle V. Neue Betrachtungen zum Krankheitsbild der primären Varikosis. Internist Prax 2002; 42: 583- 597

28 Hach W, Hach-Wunderle V. Die Rezirkulationskreise der primären Varikosis. Berlin-Heidelberg-New York: Springer, 1994: 27 - 48

29 Trendelenburg F. Ueber die Unterbindung der Vena saphena magna bei Unterschenkelvaricen. Beitr Klein Chir 1890; 7: 195-210

30 Eklöf B, Rutherford RB, Bergan JJ et al. für das Ad-hoc-Komitee für die Revision der CEAP-Klassifikation des American Venous Forum. Revision der CEAP-Klassifizierung für chronische Venenleiden. Phlebologie 2005; 34: 220- 225

31 von Uslar D, Schultz-Ehrenburg U. Falsche nicht besserbare chronischen Veneninsuffizienz. Phlebol Proktol 1988; 17: $96-100$

32 Weindorf N, Schultz-Ehrenburg U. Der Wert der Photoplethysmographie (Lichtreflexionsrheographie) in der Phlebologie. VASA 1986; 15: 397-406

33 Wienert V. Lichtreflexionsrheographie. Möglichkeiten und Grenzen. Z Hautkr 1985; 60: 1850-1854

34 Wienert V, Blazek V, Mayer O. Nichtinvasive hämodynamische Untersuchungen vor und nach Babcock-Stripping. Phlebol Proktol 1986; 15: 72 - 74

35 Wienert $V$. Anwendungsfehler und Fehlinterpretationen bei der Lichtreflexionsrheographie. Phlebol 1991; 20: 126-130

36 Blazek V, May R, Stemmer $R$ et al. Die Standardisierung der LRR-Untersuchung. In: May R, Stemmer R (Hrsg). Die Licht-Reflexionsrheographie. Erlangen: perimed, 1984: 151-155
37 Wienert V, Rütten M. Der Einfluß unterschiedlicher Temperaturen auf die venöse Hämodynamik der unteren Extremität. Phlebol Proktol 1984; 13: $25-26$

38 Bays RA, Healy DA, Atnip RG et al. Validation of air plethysmography, photoplethysmography, and duplex ultrasonography in the evaluation of severe venous stasis. IVasc Surg 1994; 20: 721 - 727

39 Kahle B, Schulze-Dirks A, Petzoldt D. Bedeutung der Duplex-Sonographie in der Diagnostik venöser Erkrankungen. Hautarzt 1993; 44: 275-281

40 Kahle B, Köhler P, Utermann S. Duplexsonographische Untersuchungen zum venösen Stromzeitvolumen in der Vena femoralis bei chronisch venöser Insuffizienz. Phlebologie 1999; 28: $81-86$

41 Labropoulos N, Leon M, Nicolaidesw AN et al. Venous reflux in patients with previous deep venous thrombosis: Correlation with ulceration and other symptoms. J Vasc Surg 1994; 20: 20 - 26

42 Lees TA, Lambert $D$. Patterns of reflux in limbs with skin changes associated with chronic venous insufficiency. Br J Surg 1993; 80: 725-728

43 Libertiny $G$, Hands L. Lower limb deep venous flow in patients with peripheral vascular disease. J Vasc Surg 1999; 29: 1065-1070

44 Weingarten MS, Branas CC, Czeredarczuk M et al. Distribution and quantification of venous reflux in lower extremity chronic venous stasis disease with duplex scanning. J Vasc Surg 1993; 18: $753-759$

45 Weingarten MS, Czeredarczuk M, Scovell S et al. A correlation of air plethysmography and color-flow-assisted duplex scanning in the quantification of chronic venous insufficiency. J Vasc Surg 1996; 24: 750 - 754

46 Jeanneret C, Aschwanden M, Labs KH et al. Duplex ultrasound for the assessment of venous reflux. Curr Probl Dermatol 1999; 27: 96 - 101

47 Masuda EM, Kistner RL. Prospective comparison of duplex scanning and descending venography in the assessment of venous insufficiency. Am J Surg 1992; 164: $254-259$

48 Rodriguez AA, Whitehead CM, McLaughlin RL et al. Duplex-derived valve closure times fail to correlate with reflux flow volumes in patients with chronic venous insufficiency. J Vasc Surg 1996; 23: 606-610

49 van Bemmelen PS, Beach K, Bedford G et al. The mechanism of venous valve closure. Its relationship to the velocity of reverse flow. Arch Surg 1990; 125: $617-619$

50 Simonian SJ. Recurrence following combined surgery and postoperative sclerotherapy of varicose veins. In: Negus $D$, Jantet $G$, Coleridge-Smith PD (eds). Phlebology 1995. Berlin: Springer, 1995: 474-476

51 Partsch H. Konservative Therapie venöser Leiden. Acta Chir Austriaca 2000; 32 (Suppl. 164) 5: 22-26

52 Benkö T, Kalik I, Chetty MN. The physiological effect of compression stockings on bloos flow in the lower limb: An Assessment with Colour Doppler ultrasoud. Phlebology 1999; 14: 17-20

53 Buhs CL, Bendick PJ, Glover IL. The effect of graded compression stockings on the lower leg venous system during daily activity. J Vasc Surg 1999; 30: $830-835$

54 Ibegbuna V, Delis KT, Nicolaides AN et al. Effect of elastic compression stockings on venous hemodynamics during walking. JVasc Surg 2003; 37: 420 425

55 Jünger M, Sippel K. Kompressionstherapie bei chronischer venöser Insuffizienz. Neue Prüfverfahren und therapeutische Optionen. Hautarzt 2003; 54: $1045-1052$

56 Kahle B, Bolz S, Feise K. Quantitative Analyse des Einflusses von standardisiertem Gehen auf die venöse Hämodynamik bei postthrombotischem Syndrom. Phlebologie 2002; 31: 126-131

57 Partsch H, Menzinger G, Borst-Krafek B et al. Does thigh compression improve venous hemodynamics in chronic venous insufficiency? J Vasc Surg 2002; 36: 948 - 952

58 Partsch $H$, Menzinger $G$, Mostbeck A. Inelastic leg compression is more effective to reduce deep venous refluxes than elastic bandages. Dermtol Surg 1999; 25: 695-700

59 Hafner HM, Eichner M, Jünger M. Medizinsche Kompressionstherapie. Zentalbl Chir 2001; 126: 551-556

60 Kahle B, Idzko M, Norgauer J et al. Tightening Tight Junctions with Compression Therapy. J Invest Derm 2003; 121: 1228-1229

61 Martinez M], Bonfill X, Moreno RM et al. Phlebotonics for venous insufficiency. Cochrane Database of Systematic Reviews 2005, Issue 3. Art. No.: CD003229. DOI: 10.1002/14651858.CD003229.pub2

62 Mollard JM, Boissier C. medical treatment of chronic venous insufficiency. Rev Prat 1994; 44: $763-768$

63 Ramelet AA, Monti M. Phlebology - the guide. 4th ed. Paris: Masson, 1999

64 Hamel-Desnos C, Desnos P, Wollmann JC et al. Evaluation of the efficacy of Polidocanol in the form of foam compared with liquid form in sclerothera- 
py of the long saphenous vein: Initial results. Dermatol Surg 2003; 29: $1170-1175$

65 Rabe E, Pannier F, Gerlach $\mathrm{H}$ et al. Leitlinie: Sklerosierungsbehandlung der Varikosis. Phlebologie 2008; 37: 27-34

66 Cabrera J, Redondo P, Becerra A et al. Ultrasound-guided injection of Polidocanol microfoam in the management of venous leg ulcers. Arch Dermatol 2004; 140: 667-673

67 Stücker M, Reich S, Hermes N, Altmeyer P. Safety and efficacy of perilesional sclerotherapy in leg ulcer patients with postthrombotic syndrome and/or oral anticoagulation with phenprocoumon. J Dtsch Dermatol Ges 2006; 4: $734-738$

68 Wildenhus B. Endovenöse kathetergestützte Schaumsklerosierung. Phlebologie 2005; 34: $165-170$

69 Brodersen JP. Catheter-assisted vein sclerotherapy: A new approach for sclerotherapy of the greater saphenous vein with double-lumen balloon catheter. Deramtol Surg 2007; 33: 469-475

70 Babcock WW. A new operation for the extirpation of varicose veins. New York Med J 1907; 23: 153-156

71 Hach W, Hach-Wunderle V. Das Stripping und die Konkurrenzverfahren zur chirurgischen Behandlung der Stamm-Varikosis. Gefäßchirurgie 2000; 5: 56-61

72 Lacroix $H$, Nevelsteen A, Suy $R$. Invagination versus classic stripping of the long saphenous vein. A randomized prospective study. Acta Chir Belg 1999; 99: 22-25

73 Oesch A. „Pin-stripping“ a novel method of atraumatic stripping. Phlebology 1993; 8: $171-178$

74 Friedl PG, Rappold EM. Kryostripping der V saphena magna/parva - ein etabliertes Verfahren. Klinische Langzeitergebnisse. Vasomed 1998; 10 : 24

75 Francesci $C$. Théorie et pratique de la cure conservatrice et hémodynamique de l'insuffisance veineuse en ambulatoire. Precy-sous-Thil: Armoncon, 1988

76 Proebstle TM, Krummenauer F, Gül D et al. Nonocclusion and early reopening of the great saphenous vein after endovenous laser treatment is fluencedependent. Derm Surg 2004; 30: 174-178

77 Pichot O, Kabnick LS, Creton $D$ et al. Duplex ultrasound scan findings two years after great saphenous vein radiofrequency endovenous obliteration. IVasc Surg 2004; 39: 189- 195

78 Lurie F, Creton D, Eklof B et al. Prospective randomised Study of endovenous radiofrequency (Closure) versus Ligation and Vein Strippiung (EVOLVeS): Two-year Follow-up. Eur J Endovas Surg 2005; 29: 67-63

79 Geier B, Voigt I, Barbera L, Marpe B et al. Extraluminale Valvuloplastie bei Stamminsuffizienz der V. saphena magna. Fünf-Jahres-Ergebnisse. Phlebologie 2004; 33: 149-155

\section{CME-Fragen Diagnostik und Therapie der Varikosis}

Welche Aussage zur Epidemiologie der Varikosis trifft nicht zu?

A Die Häufigkeit von Venenerkrankungen im Allgemeinen ist in der Tübinger Studie vergleichbar zur Bonner Venenstudie.

B In der Tübinger Studie litten 13\% der untersuchten Personen an einer fortgeschrittenen chronischen venösen Insuffizienz.

C In der Bonner Venenstudie litten 3,6\% der Probanden an fortgeschrittenen Stadien der CVI mit trophischen Störungen an der Haut bis hin zum Ulcus cruris.

D Die Bonner Studie zeigte in 14,3\% der Fälle ausgeprägte Varizen ohne die typischen Zeichen einer CVI.

E Die Bonner Studie zeigte in 9,6\% der Fälle ausgeprägte Varizen ohne die typischen Zeichen einer CVI.

2 Welche Aussage trifft zu?

A Die Stadieneinteilung der Stammvarikosis nach Hach entspricht der Schwere des Krankheitsbildes.

B Die Stadieneinteilung der Stammvarikosis nach Hach betrifft lediglich die Refluxlänge.

c Die Stadieneinteilung der Stammvarikosis nach Hach betrifft lediglich die Refluxdauer.

D Die Stammvarikosis der V. saphena magna lässt sich inspektorisch in unterschiedliche Stadien einteilen.

E Die Stammvarikosis der V. saphena parva spielt klinisch keine Rolle.

A Für die Entwicklung einer chronischen venösen Insuffizienz ist ein postthrombotischer Zustand Voraussetzung.

B Ein postthrombotisches Syndrom führt immer zu einer chronischen venösen Insuffizienz.

C Die ambulatorische venöse Hypertonie ist relevant für die Ausbildung trophischer Störungen.

D Die Rezirkulationskreise sind pathophysiologisch bedeutungslos.

E International ist derzeit die Stadieneinteilung der CVI nach Widmer gebräuchlich.

Welcher Faktor spielt in der Ätiologie der Varikosis keine Rolle?

A hämodynamische Aspekte

B endotheliale Faktoren

C lysosomale Enzyme

D genetische Dispostion

E Kollagenosen 
5 Welche Aussage zur Diagnostik trifft zu?

A Die Phlebografie ist in der präoperativen Diagnostik obligat.

B Die Dopplersonografie ist eine ausreichende diagnostische Methode für die Planung des operativen Vorgehens.

C Präoperativ muss immer eine Funktionsdiagnostik mittels DPPG durchgeführt werden.

D Die Duplexsonografie erlaubt die gleichzeitige morphologische und funktionelle Diagnostik.

E Die Venenverschlussplethysmografie kann die Dopplersonografie ersetzen.

6 Welcher hämodynamische Parameter lässt sich mittels Duplexsonografie erheben?

A Refluxdauer

B Refluxvolumen

C arterielles Stromzeitvolumen

D venöse Blutflussgeschwindigkeit

E alle genannten Parameter

\section{Welche Aussage trifft nicht zu?}

A Die Kompressionstherapie ist hämodynamisch wirksam.

B Unelastische Kompressionsmaterialien sind effektiver in der Refluxbehandlung als elastische Materialien.

c Kompressionsstrümpfe unterschiedlicher Kompressionsklassen unterscheiden sich in der Länge.

D Medizinische Kompressionsstrümpfe sind für die Mikrozirkulation bedeutsam.

E Venentherapeutika zur Ödemreduktion können die Kompressionstherapie nicht ersetzen.
8 Welche Aussage ist falsch? Absolute Kontraindikationen der Sklerosierungstherapie sind: bekannte Allergie auf das Sklerosierungsmittel längerfristige Immobilität oder Bettlägerigkeit lokale, im Bereich der Sklerosierung gelegene oder schwere generalisierte Infektionen

D Dauerantikoagulation mit Cumarinen

E fortgeschrittene arterielle Verschlusskrankheit im Stadium III oder IV

9 Welche Aussage ist falsch? Potenzielle Nebenwirkungen und Komplikationen der Schaumsklerosierung sind: Matting

passagerer Hörverlust

Thrombosen

Sehstörungen

Hyperpigmentierungen

\section{0}

A Für die endoluminale Lasertherapie spielt die Energiedosis eine entscheidende Rolle.

B Die Wellenlänge ist in der endoluminalen Lasertherapie nicht bedeutsam.

C Die Radiowellenobliteration kann nur zur Therapie der V. saphen magna eingesetzt werden.

D Der Erfolg der Radiowellenobliteration ist Fluenceabhängig.

E Die Schaumsklerosierungstherapie kann nicht in einer Sitzung mit der endoluminalen Lasertherapie durchgeführt werden.

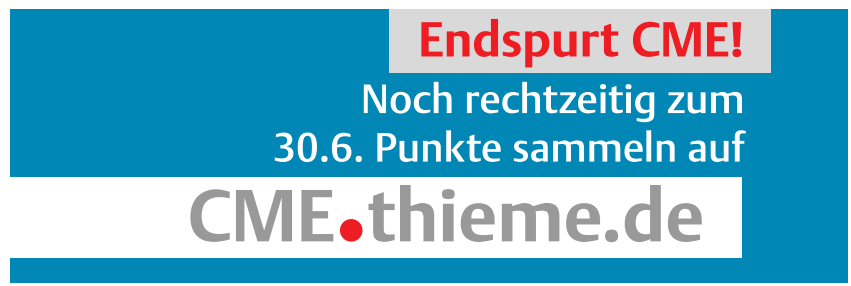


A Angaben zur Person

Name, Vorname, Titel:
Straße, Hausnr.:

Anschrift: privat dienstlich

EFN-Nummer:

Ich bin Mitglied der Ärztekammer (bitte Namen der Kammer eintragen):

Jahr meiner Approbation:

Ich befinde mich in der Weiterbildung zum:

Ich habe eine abgeschlossene Weiterbildung in (bitte Fach eintragen):

Ich bin tätig als: Assistenzarzt Oberarzt Chefarzt niedergelassener Arzt Sonstiges:

\section{B Lernerfolgskontrolle}

Bitte nur eine Antwort pro Frage ankreuzen

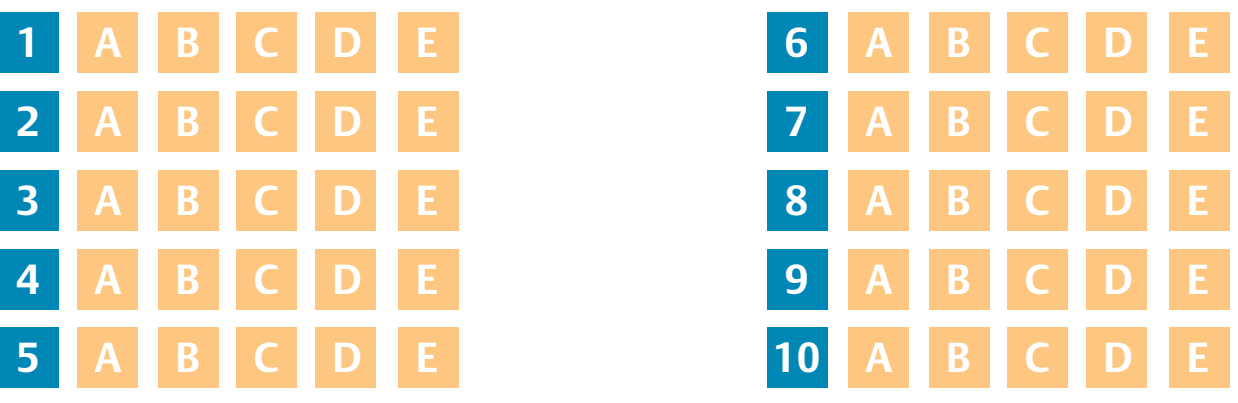

Erklärung

Ich versichere, dass ich die Beantwortung der Fragen selbst und ohne Hilfe durchgeführt habe

Ort | Datum:

D Feld für CME-Wertmarke

Bitte in dieses Feld die CME-Wertmarke kleben oder Ihre Abonnement-Nummer eintragen: (siehe Adressaufkleber)

Zertifizierungsfeld (wird durch den Verlag ausgefüllt)
Unterschrift:

\section{E Zertifizierungsfeld}

\section{Ihr Ergebnis}

Sie haben $\quad$ von 10 Fragen richtig beantwortet.

Sie haben

bestanden und 3 CME-Punkte erworben.

nicht bestanden

Stuttgart, den

Datum

Stempel/Unterschrift

Bitte unbedingt Rückseite ausfüllen! 


\section{F Fragen zur Zertifizierung}

Eine Antwort pro Frage. Bitte unbedingt ausfüllen bzw. ankreuzen, da die Evaluation sonst unvollständig ist!

\section{Didaktisch-methodische Evaluation}

1 Das Fortbildungsthema kommt in meiner ärztlichen Tätigkeit

häufig vor

regelmäßig vor selten vor gar nicht vor

2 Zum Fortbildungsthema habe ich

eine feste Gesamtstrategie

noch offene Einzelprobleme:

keine Strategie

3 Hinsichtlich des Fortbildungsthemas

fühle ich mich nach dem Studium des Beitrags in meiner Strategie bestätigt

habe ich meine Strategie verändert:

habe ich erstmals eine einheitliche Strategie erarbeitet

habe ich keine einheitliche Strategie erarbeiten können

4 Wurden aus der Sicht Ihrer täglichen Praxis heraus wichtige Aspekte des Themas nicht erwähnt?

zu knapp behandelt?

überbewertet?

\begin{tabular}{|l|l|}
\hline ja, welche \\
\hline ja, welche \\
\hline ja, welche \\
\hline
\end{tabular}

5 Verständlichkeit des Beitrags

Der Beitrag ist nur für Spezialisten verständlich

Der Beitrag ist auch für Nicht-Spezialisten verständlich

6 Beantwortung der Fragen

Die Fragen lassen sich aus dem Studium des Beitrages allein beantworten

Die Fragen lassen sich nur unter Zuhilfenahme zusätzlicher Literatur beantworten

7 Die Aussagen des Beitrages benötigen eine ausführlichere Darstellung

zusätzlicher Daten

von Befunden bildgebender Verfahren

die Darstellung ist ausreichend

8 Wie viel Zeit haben Sie für das Lesen des Beitrages und die Bearbeitung des Quiz benötigt?

Einsendeschluss 15.6. 2010
Bitte senden Sie den vollständigen Antwortbogen zusammen mit einem an Sie selbst adressierten und ausreichend frankierten Rückumschlag an den

Georg Thieme Verlag KG, Stichwort „CME“, Postfach 3011 20, 70451 Stuttgart 